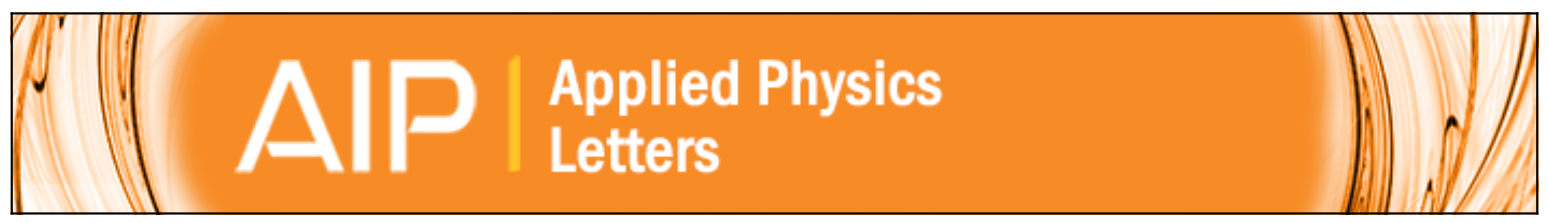

\title{
Underlying mechanism of domain-wall motions in soft magnetic thin-film nanostripes beyond the velocity-breakdown regime
}

Sang-Koog Kim, Jun-Young Lee, Youn-Seok Choi, Konstantin Yu. Guslienko, and Ki-Suk Lee

Citation: Applied Physics Letters 93, 052503 (2008); doi: 10.1063/1.2968138

View online: $\mathrm{http}: / / \mathrm{dx}$. doi.org/10.1063/1.2968138

View Table of Contents: http://scitation.aip.org/content/aip/journal/apl/93/5?ver=pdfcov

Published by the AIP Publishing

\section{Articles you may be interested in}

$360^{\circ}$ domain wall injection into magnetic thin films

Appl. Phys. Lett. 103, 222404 (2013); 10.1063/1.4828563

Mechanism of reversing the Neel domain walls in the Co nanostripes with transverse magnetic anisotropy Appl. Phys. Lett. 101, 252412 (2012); 10.1063/1.4772981

Field- and current-induced domain-wall motion in permalloy nanowires with magnetic soft spots

Appl. Phys. Lett. 98, 202501 (2011); 10.1063/1.3590267

Thermally activated domain wall dynamics in a disordered magnetic nanostrip

J. Appl. Phys. 109, 07D345 (2011); 10.1063/1.3565402

Gyrotropic linear and nonlinear motions of a magnetic vortex in soft magnetic nanodots Appl. Phys. Lett. 91, 132511 (2007); 10.1063/1.2783272

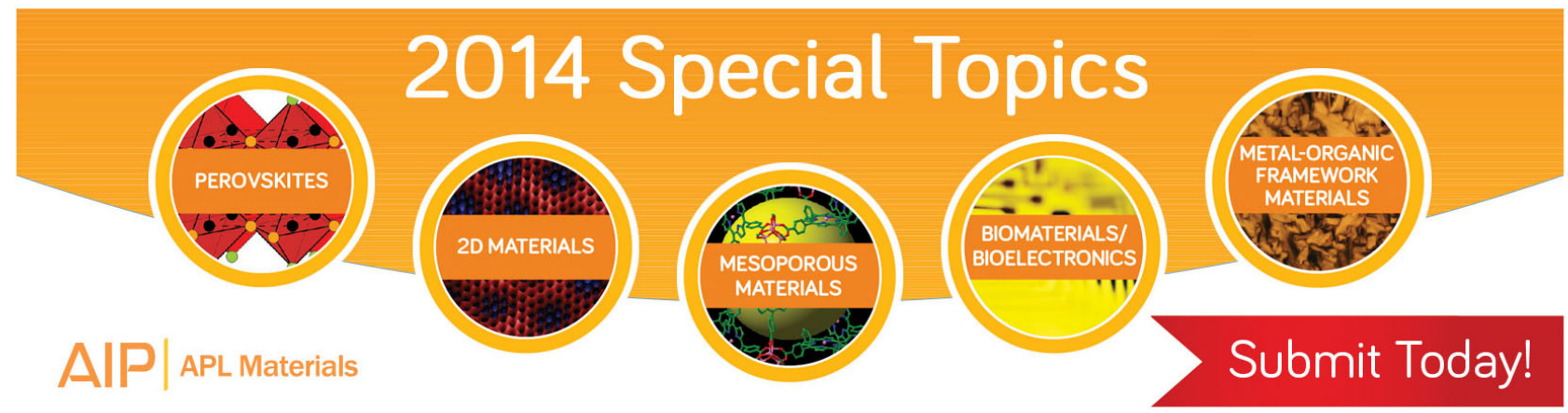




\title{
Underlying mechanism of domain-wall motions in soft magnetic thin-film nanostripes beyond the velocity-breakdown regime
}

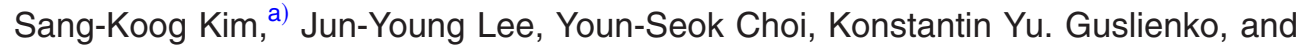 \\ Ki-Suk Lee \\ Research Center for Spin Dynamics \& Spin-Wave Devices and Nanospinics Laboratory, \\ Department of Materials Science and Engineering, College of Engineering, Seoul National University, \\ Seoul 151-744, Republic of Korea
}

(Received 27 May 2008; accepted 17 July 2008; published online 6 August 2008)

\begin{abstract}
It is known that oscillatory domain-wall (DW) motions in soft magnetic thin-film nanostripes above the Walker critical field lead to remarkable reductions in the average DW velocities. In a much-higher-field region beyond the velocity-breakdown regime, however, the DW velocities have been found to increase in response to a further increase of the applied field. We report on the physical underlying mechanism of this unexpected behavior. We associate the mechanism with the serial dynamic processes of the nucleation of vortex-antivortex pairs inside the stripe or at its edges, the nonlinear gyrotropic motions of vortices and antivortices, and their annihilation process. Moreover, this work evidences that a two-dimensional soliton model is required for adequate interpretation and understanding of DW motions in the linear- and oscillatory-DW-motion regimes as well as in the beyond-velocity-breakdown regime. (C) 2008 American Institute of Physics. [DOI: 10.1063/1.2968138]
\end{abstract}

Magnetic field driven magnetization (M) reversals in bulk or thin-film magnetic materials via domain-wall (DW) movements as well as coherent or incoherent $\mathbf{M}$ rotation have been historically longstanding issues in the research field of magnetism. ${ }^{1}$ The motion of a head-to-head (or tailto-tail) DW in magnetic thin-film nanostripes under applied magnetic fields and/or spin-polarized current has been a subject of cutting-edge researches, ${ }^{2-10}$ and has very promising potential applications to new classes of race-track memory ${ }^{11}$ and logic ${ }^{12}$ devices. When a static field $H$ is applied to a DW, its velocity increases with increasing $H$ in a low- $H$ region, but remarkably drops above a certain critical field known as the Walker field $H_{w}{ }^{2-4,13-15}$ From previous studies ${ }^{16,17}$ it is known that in the low- $H$ region a single transverse wall (TW) moves steadily along the nanostripe parallel to the field direction. The velocity breakdown is known to be associated with the oscillations of the internal structure of a single moving DW between the TW and the vortex or antivortex wall (VW or AVW) (Ref. 16) with a time period $T=2 \pi / \gamma H{ }^{17}$ where $\gamma$ is the gyromagnetic ratio. In the velocity-breakdown region, a $\mathrm{V}(\mathrm{AV})$ within the $\mathrm{VW}(\mathrm{AVW})$ exhibits a nonlinear gyrotropic motion in the nanostripe, resulting in the reduced average DW velocity $\bar{v}$ due to the motions of $\mathrm{V}$ and $\mathrm{AV}$ against the $\mathbf{M}$ reversal direction.

In a much-higher-field region beyond the velocitybreakdown regime, on the contrary the average DW velocity has been observed to again increase with a further increase of $H{ }^{4,16}$ The physical origin and detailed understanding of this unexpected behavior remains elusive, whereas the linear and oscillatory DW dynamics have been described by onedimensional (1D) models ${ }^{13,18}$ and/or a two-dimensional (2D) dynamic soliton model. ${ }^{16,17}$

In this letter, we report on the dynamic mechanism of the increased DW velocities in the higher- $H$ region beyond the

\footnotetext{
${ }^{\text {a) }}$ Author to whom all correspondence should be addressed. Electronic mail: sangkoog@snu.ac.kr.
}

DW oscillatory regime, which is as yet unknown. The underlying mechanisms found from this work are associated with the serial processes of the nucleation, gyrotropic motion, and annihilation of $\mathrm{V}$-AV pairs, while maintaining the conservation of their total topological charge in the nanostripes. The present results offer a new and deeper physical insight in $\mathrm{V}-\mathrm{AV}$-mediated $\mathbf{M}$ reversal dynamics in nanostripes beyond the velocity-breakdown regime. Moreover, we confirm that a $2 \mathrm{D}$ dynamic soliton model previously proposed ${ }^{16,17}$ is necessary for adequate physical descriptions/interpretations of DW dynamics mediated via the nucleation, gyrotropic motion, and annihilation of magnetic solitons of $\mathrm{Vs}_{\mathrm{s}}$ and $\mathrm{AVs}$ in 2D systems such as thin-film nanostripes, covering all the different $H$ strength regimes.

In the present micromagnetic simulations, we used a rectangular-shaped soft magnetic nanostripe of $10 \mathrm{~nm}$ thickness, $6 \mu \mathrm{m}$ length, and different widths $w=140$ and $240 \mathrm{~nm}$. The material parameters used are as follows: the saturation magnetization $M_{s}=8.6 \times 10^{5} \mathrm{~A} / \mathrm{m}$, the exchange constant $A$ $=1.3 \times 10^{-11} \mathrm{~J} / \mathrm{m}$, and zero magnetocrystalline anisotropy, corresponding to the values of permalloy $(\mathrm{Py})$. The simulation procedure and details were described elsewhere. ${ }^{16}$ The initial DW type used in this study was a head-to-head TW having nonzero average transverse $\mathbf{M}$ as the ground state at $H=0$ in the given nanostripe [Fig. 1(a)]. This DW was driven by static magnetic fields of various strengths applied along the long axis of the nanostripes (in the $+x$ direction). The $\mathbf{M}$ reversal dynamics for $w=140 \mathrm{~nm}$ and with $H=5,25$, and 150 Oe are represented by the displacements of DW versus time ( $D$ vs $t$ ) curves shown in Fig. 1(b). Those three different field strengths were selected from the represented three distinct $H$ regions, denoted as regions I, II, and III, corresponding to $H<H_{W}, H_{W}<H<H_{\text {mult }}$, and $H>H_{\text {mult }}$, respectively. $H_{\text {mult }}$ represents a field strength above which a multi-V-AV state appears during the $\mathbf{M}$ reversal, unlike a single DW motion in regions I and II whether it experiences a steady motion or periodic transformation. In this geometry, the esti- 
(a)
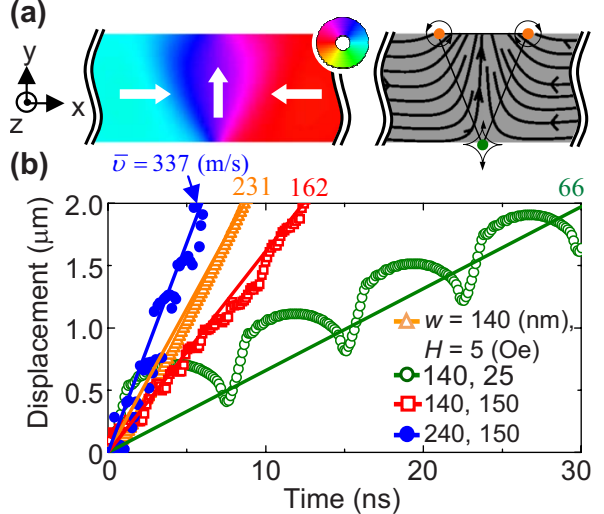

FIG. 1. (Color online) (a) Left: In-plane M configuration of a TW with the nonzero transverse ( $+y$ direction) $\mathbf{M}$ component in the nanostripe. The colors denote the local in-plane $\mathbf{M}$, as indicated by the color wheel. Right: Representation of half-integer topological charges located at both stripe edges, along with the nucleation sites for $\mathrm{V}$ (dark yellow dots) and AV (green dot). The streamlines are along the in-plane orientation of the local M. (b) DW displacement vs time curves, for DW motions driven by magnetic fields $H$ along long axis of nanostripe of $10 \mathrm{~nm}$ thickness, $6 \mu \mathrm{m}$ length, and two different widths of 140 and $240 \mathrm{~nm}$.

mated values of $H_{W}$ and $H_{\text {mult }}$ were $\sim 10$ and $\sim 90 \mathrm{Oe}$, respectively.

The characteristic $D$ vs $t$ curves in the represented different $H$ regions show that the different speeds of the $\mathbf{M}$ reversals are related closely to their quite different governing mechanisms. In region III $(H=150 \mathrm{Oe})$, beyond the velocitybreakdown regime, the $\mathbf{M}$ reversal becomes faster $(\bar{v}$ $=162 \mathrm{~m} / \mathrm{s})$ than that $(\bar{v}=66 \mathrm{~m} / \mathrm{s})$ in region II as well as more or less comparable to that $(\bar{v}=231 \mathrm{~m} / \mathrm{s})$ in region I. As another example, the $D$ vs $t$ curve for $w=240 \mathrm{~nm}$ and $H$ $=150$ Oe also shows that the reduced $\bar{v}$ in region II is markedly increased to $\bar{v}=337 \mathrm{~m} / \mathrm{s}$ in region III. Although the physical origin and detailed mechanism of DW motion dynamics in regions I and II (see Refs. 16 and 17) have been clarified, the underlying reversal mechanism in region III has not been known yet.

To find the exact $\mathbf{M}$ reversal mechanism in region III, we display the serial snapshot images taken at the indicated times for the temporal evolution of the head-to-head DW for the cases of $w=140 \mathrm{~nm}$ and $H=150$ Oe in Fig. 2(a), and for the case of $w=240 \mathrm{~nm}$ and $H=100$ Oe in Fig 2(b). Unlike the motions in regions I and II, ${ }^{16,17}$ the DW motion in region III is neither a steady motion of a TW nor a periodic oscillation of the internal structure of a single moving DW between its different types (TW, VW, or AVW). Rather, the internal structure of the moving DW contains several magnetic topological solitons ${ }^{16,17}$ such as Vs and AVs whose cores are represented as red or blue spots according to the $\mathbf{M}$ orientation of their cores. Those serial snapshot images display the individual steps associated with the V-AV-pair mediated dynamic processes.

To elucidate the characteristic dynamic features of the nucleation, gyrotropic motion, and annihilation of the V-AV pairs, we show their zoomed images along with schematic illustrations in Fig. 3. For convenience, hereafter, we denote Vs and AVs by the symbols $V$ and $\bar{V}$, respectively, and their core $\mathbf{M}$ orientations by the arrows $\uparrow$ and $\downarrow$ in the symbols' subscripts, respectively. Case I, shown in Fig. 3(a), illustrates that the $\mathrm{V}$ and $\Delta \mathrm{V}$ with the same up-core orientation nucleate (a)

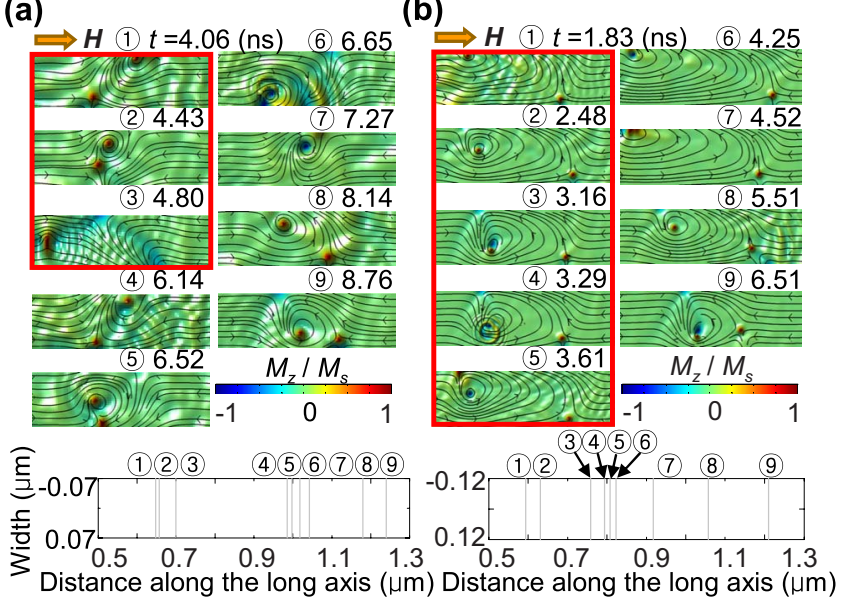

FIG. 2. (Color online) Serial snapshot images of the temporal dynamic evolution of DW motions in a given nanostripe of $w=140 \mathrm{~nm}$ and with $H$ $=150 \mathrm{Oe}$ in (a) and $w=240 \mathrm{~nm}$ and $H=100 \mathrm{Oe}$ in (b). The field direction is marked by the yellow colored arrow. The different colors and streamlines indicate the normalized out-of-plane $\mathbf{M}$ components, as noted by the color bar, and the in-plane $\mathbf{M}$ orientation, respectively. The red and blue spots indicate the up and down core orientations, respectively. The positions of the moving DWs at indicated times are noted by numbered images.

(1) at the opposite edges of the nanostripe at nearly the same time. Then, both the $\mathrm{V}$ and $\mathrm{AV}$ move inward the nanostripe, become closed (2), according to their own nonlinear gyrotropic motions, and then finally collapse (3) inside the nanostripe and annihilate [see the schematic core positions (right panel)]. Immediately after the V-AV annihilation, strong spin waves are emitted (3) according to the mechanism reported earlier. ${ }^{19}$ On the other hand, case II, for $w$ $=240 \mathrm{~nm}$ and $H=100 \mathrm{Oe},{ }^{20}$ is displayed in Fig. 3(b), where $V_{\uparrow}$ and $\bar{V}_{\uparrow}$ simultaneously nucleate (1) at the opposite edges as in case I. However, unlike case I, the $V_{\uparrow}$ moves (2) inward faster than $\bar{V}_{\uparrow}$, while the $\bar{V}_{\uparrow}$ is maintained near the same stripe edge. As the next step, the $V_{\uparrow}$ moves further inward, and around it an additional $V_{\downarrow}^{\prime}-\bar{V}_{\downarrow}^{\prime}$ pair with down core orientation is nucleated (3)). The prime in the superscripts is used to distinguish the newly nucleated V-AV pair from the $\mathrm{V}$-AV pair $\left(V_{\uparrow}-\bar{V}_{\uparrow}\right)$ nucleated earlier at both nanostripe

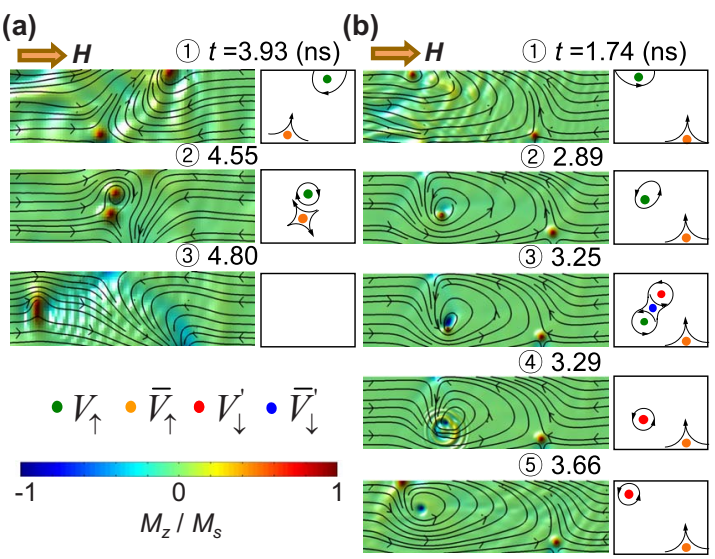

FIG. 3. (Color online) Snapshot images (left) and schematic illustrations (right) representing the nucleation, gyrotoropic motion, and annihilation of Vs and AVs, along with spin-wave radiation. (a) and (b) correspond to the dynamic evolutions marked by the red boxes in Figs. 2(a) and 2(b), respectively. The different colors denote the different types of vortices, with the indicated core crientations as noted in the inset. 
edges. Then, the $V_{\uparrow}-\bar{V}_{\downarrow}^{\prime}$ pair annihilation process is followed (4) according to the mechanism reported in Refs. 16 and 21, and is accompanied by spin wave emission by the core reversal. ${ }^{19}$ The remaining new vortex $\left(V_{\downarrow}^{\prime}\right)$ with the reversed core orientation moves towards the up edge of the nanostripe according to its own gyrotropic motion (5), as illustrated in the schematic drawing (right panel) and the serial snapshot images in Fig. 3. Although cases I and II differ in the overall $\mathbf{M}$ reversal mechanism, the same serial processes associated with the individual steps of the nucleation, nonlinear gyrotropic motion, and annihilation of the Vs and AVs are commonly involved.

In order to gain better physical insight into those common individual processes, it is necessary to adopt the V-AVpair mediated DW dynamics with regard to the emission, motion, and absorption of topological solitons ${ }^{16,17}$ in the framework of collective dynamic variables-the soliton core positions. The in-plane $\mathbf{M}$ configuration of a single symmetric TW resembles an isosceles triangle with three apexes [Fig. 1(a), right]. An AV can nucleate only at a single apex, in this case, placed at the down stripe edge, and a $\mathrm{V}$ can nucleate at one of the double apexes at the upper edge, so that the three apexes can act as the nucleation sites for the magnetic solitons. This is so, because the $\mathbf{M}$ configuration of the moving TW can be represented by magnetic solitons of half-integer topological charges $q$ located on the stripe edges, as shown in the right of Fig. 1(a). A similar approach was developed to explain the oscillatory transformation of the internal structure of the different-type DWs in the nanostripes within the velocity-breakdown regime. ${ }^{16}$

In the higher-field region III, however, both $\mathrm{V}$ and $\mathrm{AV}$ simultaneously nucleated at the opposite edges, as seen in Fig. 3. Such nucleation, compared with that of a single V or $\mathrm{AV}$ in region II, lead to the increase of the exchange energy due to the presence of additional soliton cores. These events occur owing to a remarkable reduction of the Zeeman energy by a further increase of $H$, resulting in the excess exchange energy, due to the increased number of the soliton cores inside the nanostripe, being compensated. For the cases of further increases of $w$ and/or $H$, the number of the nucleated $\mathrm{V}-\mathrm{AV}$ pairs was observed to increase. The $\mathbf{M}$ reversals via such multi-V-AV pairs are much faster than those via a single $\mathrm{V}$ or AV motion or a single $\mathrm{V}-\mathrm{AV}$ pair mediated DW motion. Consequently, the reason for the increased DW velocities in the beyond-velocity-breakdown regime is because V-AV-pair nucleation is the dominant reversal process in such high- $H$ region. In such V-AV-pair mediated $\mathbf{M}$ dynamics, the total topological charge of all magnetic solitons (indexed by $j$ ) inside the nanostripe should be maintained constant, i.e., $\sum_{j} q_{j}=$ const. For the case of this nanostripe geometry, the constant value is zero because the initial DW structure is a TW that has half integer charges $q=+1 / 2$ and $-1 / 2$ at the opposite edges. The $\mathrm{V}$ and $\mathrm{AV}$ also bear an integer topological charges $q=1$ and $q=-1$, respectively. ${ }^{17}$

In conclusion, beyond the velocity-breakdown regime the $\mathbf{M}$ reversal dynamics are a consequence of the serial dynamic processes of the nucleation, gyrotropic motion, and annihilation of coupled Vs and AVs inside or at the edges of nanostripes, which processes repeatedly take place to complete the $\mathbf{M}$ reversal. Thereby, the $\mathbf{M}$-reversal velocity in this region is determined by the nucleation process of V-AV pairs, which is the dominant reversal process, followed by the gyrotropic motions of the individual $\mathrm{V}$ and $\mathrm{AV}$, and their annihilation. Such nucleation of V-AV pairs can occur at several local regions for further increase of $H$, in order to increase the number of $\mathrm{V}-\mathrm{AV}$ pairs, making the $\mathbf{M}$ reversal much faster than that in region II. Nucleation of the coupled V-AV pairs, their motions, and annihilation play a crucial role in M-reversal dynamics in restricted geometry. The present results reflect the fact that even though 1D models can, albeit incompletely, explain linear and oscillatory DW motions in low- and intermediate-field regions, they cannot adequately describe the nucleation, gyrotropic motion, and annihilation of V-AV pairs inside nanostripes at much high fields. For adequate physical interpretations of DW motions and transformations of the internal DW structure in regions I and II, as well as of the individual processes of the nucleation, nonlinear gyrotropic motion, and annihilation of topological magnetic solitons in soft magnetic thin-film nanostripes, a 2D soliton model should be considered.

This work was supported by Creative Research Initiatives (ReC-SDSW) of MEST/KOSEF.

${ }^{1}$ A. Hubert and R. Schafer, Magnetic Domains (Springer, Berlin, 2000)

${ }^{2}$ Y. Nakatani, A. Thiaville, and J. Miltat, Nat. Mater. 2, 521 (2003).

${ }^{3}$ D. Atkinson, D. A. Allwood, G. Xiong, M. D. Cooke, C. C. Faulkner, and R. P. Cowburn, Nat. Mater. 2, 85 (2003).

${ }^{4}$ G. S. D. Beach, C. Nistor, C. Knutson, M. Tsoi, and J. L. Erskine, Nat. Mater. 4, 741 (2005); Phys. Rev. Lett. 97, 057203 (2006).

${ }^{5}$ L. Thomas, M. Hayashi, X. Jiang, R. Moriya, C. Rettner, and S. S. P. Parkin, Nature (London) 443, 197 (2006); L. Thomas, C. Rettner, M. Hayashi, M. G. Samant, S. S. P. Parkin, A. Doran, and A. Scholl, Appl Phys. Lett. 87, 262501 (2005).

${ }^{6}$ G. Meier, M. Bolte, and R. Eiselt, Phys. Rev. Lett. 98, 187202 (2007).

${ }^{7}$ J.-Y. Lee, K.-S. Lee, and S.-K. Kim, Appl. Phys. Lett. 91, 122513 (2007).

${ }^{8}$ G. S. D. Beach, M. Tsoi, and J. L. Erskine, J. Magn. Magn. Mater. 320, 1272 (2008).

${ }^{9}$ J. Yang, C. Nistor, G. S. D. Beach, and J. L. Erskine, Phys. Rev. B 77, 014413 (2008).

${ }^{10}$ M. Hayashi, L. Thomas, R. Moriya, C. Rettner, and S. S. P. Parkin, Science 11, 209 (2008); M. Hayashi, L. Tomas, C. Rettner, R. Moriya, and S. S. P. Parkin, Nat. Phys. 3, 21 (2007); Appl. Phys. Lett. 92, 112510 (2008).

${ }^{11}$ S. S. P. Parkin, U.S. Patent No. 6,834,005 (21 December 2004); 6,898,132 (24 May 2005); 6,920,062 (19 July 2005); 7,031,178 (18 April 2006); 7,236,386 (26 June 2007); S. S. P. Parkin, M. Hayashi, and L. Thomas, Science 320, 190 (2008).

${ }^{12}$ D. A. Allwood, G. Xiong, C. C. Faulkner, D. Atkinson, D. Petit, and R. P. Cowburn, Science 309, 1688 (2005).

${ }^{13}$ N. L. Schryer and L. R. Walker, J. Appl. Phys. 45, 5406 (1974).

${ }^{14}$ Y. Nakatani, A. Thiaville, and J. Miltat, J. Magn. Magn. Mater. 290-291, 750 (2005).

${ }^{15}$ S. W. Yuan and H. N. Bertram, Phys. Rev. B 44, 12395 (1991).

${ }^{16}$ J.-Y. Lee, K.-S. Lee, S. Choi, K. Y. Guslienko, and S.-K. Kim, Phys. Rev. B 76, 184408 (2007).

${ }^{17}$ K. Y. Guslienko, J.-Y. Lee, and S.-K. Kim, e-print arXiv:cond-mat/ 0711.3680

${ }^{18}$ A. Thiaville, Y. Nakatani, J. Miltat, and N. Vernier, J. Appl. Phys. 95, 7049 (2004)

${ }^{19}$ S. Choi, K.-S. Lee, K. Y. Guslienko, and S.-K. Kim, Phys. Rev. Lett. 98, 087205 (2007).

${ }^{20}$ In this case we used the cell size of $3 \times 3 \times 10 \mathrm{~nm}^{3}$ in order to clearly investigate the nucleation of a V-AV pair near the $\mathrm{V}$ that was moved from the edge after its nucleation.

${ }^{21}$ B. Van Waeyenberge, A. Puzic, H. Stoll, K. W. Chou, T. Tyliszczak, R. Hertel, M. Fähnle, H. Brückl, K. Rott, G. Reiss, I. Neudecker, D. Weiss, C. H. Back, and G. Schütz, Nature (London) 444, 461 (2006). 